\title{
Improved Performance of cMUT with Nonuniform Membranes
}

\author{
Muhammed N. Senlik, Selim Olcum, and Abdullah Atalar \\ Electrical and Electronics Engineering Department, Bilkent University, Ankara TURKEY
}

\begin{abstract}
When capacitive micromachined ultrasonic transducers are immersed in water, the bandwidth of the device is limited by the membrane's second resonance frequency. At this frequency no mechanical power to immersion medium can be transferred. We present a membrane shape to shift the second resonance frequency to a higher value. The structure consists of a very thin membrane at the outer rim with a rigid mass at the center. The stiffness of the central region moves the second resonance to a higher frequency. This membrane configuration is shown to work better in terms of gain and bandwidth as compared to conventional uniform membranes in both transmission and reception.
\end{abstract}

\section{INTRODUCTION}

Capacitive micromachined ultrasonic transducers (cMUT) promise high bandwidth at the expense of low gain compared to their piezoelectric counterpart. When immersed in water, the bandwidth of cMUT is limited by the antiresonance frequency, $f_{a}$, of the membrane, which causes an increase in the mechanical impedance of the membrane. Mechanical loading of the immersion medium causes $f_{a}$ to shift to even smaller values [1]. Recent advances in the fabrication of the transducers [2] enabled the fabrication of different membrane configurations. These configurations are shown to bring improvement in the performance of cMUTs [3]. A nonuniform membrane geometry was first proposed by [4]. Also in a recent work [5], performance measures in terms of gain-bandwidth product has been defined and it is found that, each cMUT can be optimized in terms of gain and bandwidth. In this work, our main aim is to shift $f_{a}$ of the membrane to higher frequencies while keeping the mechanical impedance of the membrane as small as possible.

We find that a nonuniform membrane, a membrane with a rigid mass at the center, results in a higher turns ratio and shifts $f_{a}$ to higher values. Results are obtained for both uniform and nonuniform membranes with reduced electrodes during the transmit and receive modes. It is shown that a nonuniform membrane is superior in many ways compared to their uniform counterpart.

Fig. 1(a) shows a cross-section of a cMUT with a uniform membrane, where the radius and the thickness are symbolized with $a$ and $t_{m}$. On the other hand, a cMUT with a nonuniform membrane configuration can be seen in Fig. 1(b). The thin membrane carrying the central mass has a thickness of $t_{m 1}$, an outer radius of $a_{1}$ and an inner radius of $a_{2}$. The central mass has an additional thickness of $t_{m 2}$ with a radius of $a_{2}$. The gap height and the thickness of the insulator are denoted as $t_{g}$ and $t_{i}$. There is a reduced electrode at the bottom of the each membrane. The membrane material is assumed to be silicon nitride.

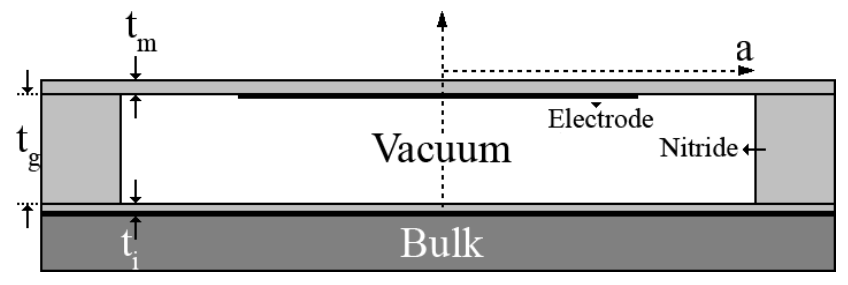

(a) cMUT with uniform membrane.

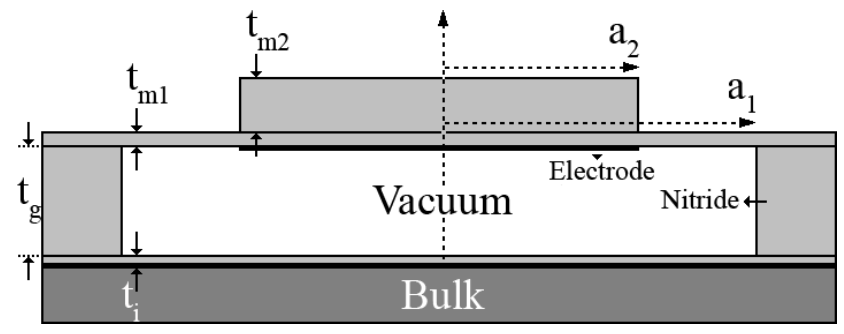

(b) cMUT with nonuniform membrane.

Fig. 1. Cross sectional view of a cMUT with a (a) uniform membrane (b) nonuniform membrane.

The Mason's equivalent circuit seen in Fig. 2 is used to model a cMUT. In the transmitter configuration, cMUT is excited by a voltage source $\left(V_{S}\right)$ to drive the acoustic impedance of the medium $\left(Z_{a} S\right)$, whereas in the receiver configuration, it is excited by the acoustical source $\left(F_{S}, Z_{a} S\right)$ driving the electrical load resistance $\left(R_{S}\right) . S$ is the area of the transducer. All equivalent circuit parameters are obtained by finite element method simulations using ANSYS following the procedures described in [6]. The material parameters used in the simulations can be found in Table I.

\begin{tabular}{ccc}
\hline Parameter & $\mathrm{Si}_{3} \mathrm{~N}_{4}$ & $\mathrm{Si}$ \\
\hline Young's Modulus & $320 \mathrm{GPa}$ & $169 \mathrm{GPa}$ \\
Density & $3270 \mathrm{~kg} / \mathrm{m}^{3}$ & $2332 \mathrm{~kg} / \mathrm{m}^{3}$ \\
Poisson's Ratio & 0.263 & 0.278 \\
Relative Permittivity & 5.7 & 11.8 \\
\hline
\end{tabular}

TABLE I

CONSTANT PARAMETERS USED IN THE SIMUlations.

\section{EFFECT OF NONUNIFORM REGION}

The modal shapes of a uniform membrane at its first natural resonance frequency, $f_{r}$, and at the second one, $f_{a}$, can be 


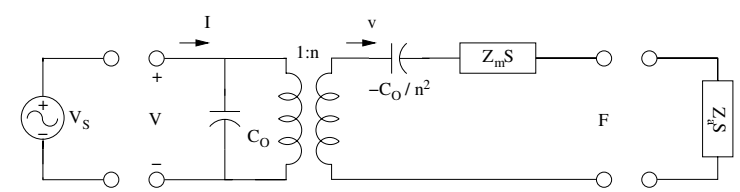

(a) Transmitter cMUT.

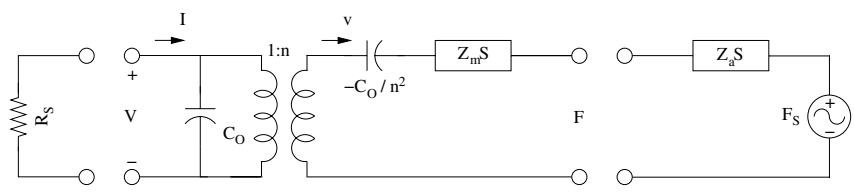

(b) Receiver cMUT.

Fig. 2. Mason model for cMUT used in the (a) transmitter configuration (b) receiver configuration.

seen in Fig. 3(a). At $f_{a}$ no acoustic power can be coupled to immersion medium. To shift $f_{a}$ to higher values, it is possible to add additional mass to deflection points. In addition, since all points on the mass move with the same velocity as a piston transducer, such a membrane shape gives higher turns ratio, $n$. The modal shapes of such a nonuniform membrane can be seen in Fig. 3(b).

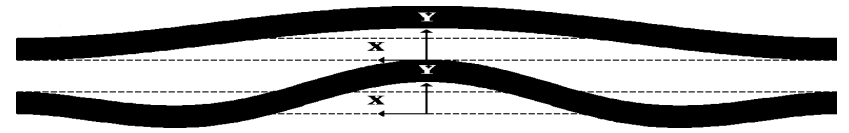

(a) Uniform membrane.

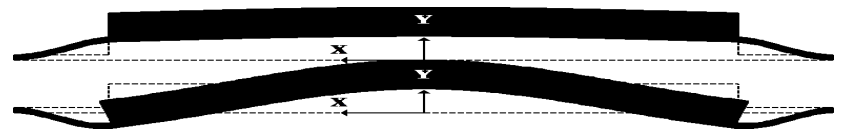

(b) Nonuniform membrane.

Fig. 3. Cross sectional view of the modal shapes of a (a) uniform membrane (b) nonuniform membrane at $f_{r}$ (upper), and $f_{a}$ (lower).

The effect of the nonuniform region on $f_{r}$ can be understood better when a first order model, a series combination of a spring and a mass, is considered. In such a model, $Z_{m}$ is given as:

$$
Z_{m}=j \omega m-\frac{j k}{\omega}
$$

with $f_{r}$,

$$
f_{r}=\frac{1}{2 \pi} \sqrt{\frac{k}{m}}
$$

where $m$ and $k$ are the mass and the stiffness of the membrane, respectively. The variation in $f_{r}$ can be seen in Fig. 4(a) obtained by keeping $a_{1}$ and $t_{m 1}$ constant, while changing $t_{m 2}$ for various values of $a_{2}$. We see that $f_{r}$ goes up initially, since the ruling effect is the stiffness of the membrane. But as the central mass gets thicker, $f_{r}$ begins to decrease as the increase in mass dominates the stiffening of membrane. This phenomenon results in a cMUT to have the same resonance frequency for two different thicknesses, which we call the first and second solutions. As $a_{2}$ is increased the maximum $f_{r}$ that can be obtained also increases. The same behavior is obtained for $f_{a}$ except the decrease in the resonance frequency starts at a higher $t_{m 2}$ (Fig. 4(b)). Although $t_{m 2}$ shifts $f_{a}$ to higher values by increasing the mass, $m$, of the membrane, referring to Eq. $1, Z_{m}$ also increases with $t_{m 2}$, which is important since it limits the bandwidth of the device.

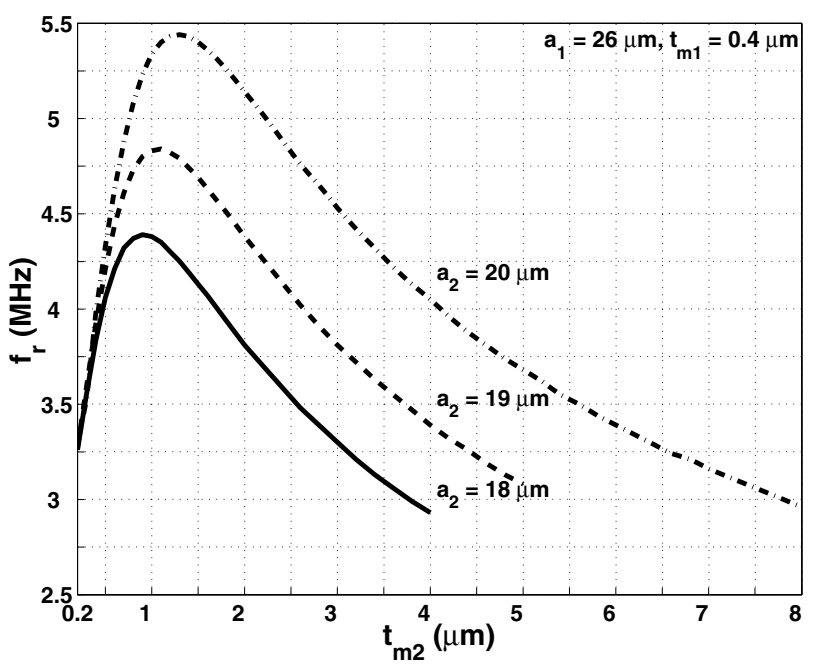

(a) $f_{r}$

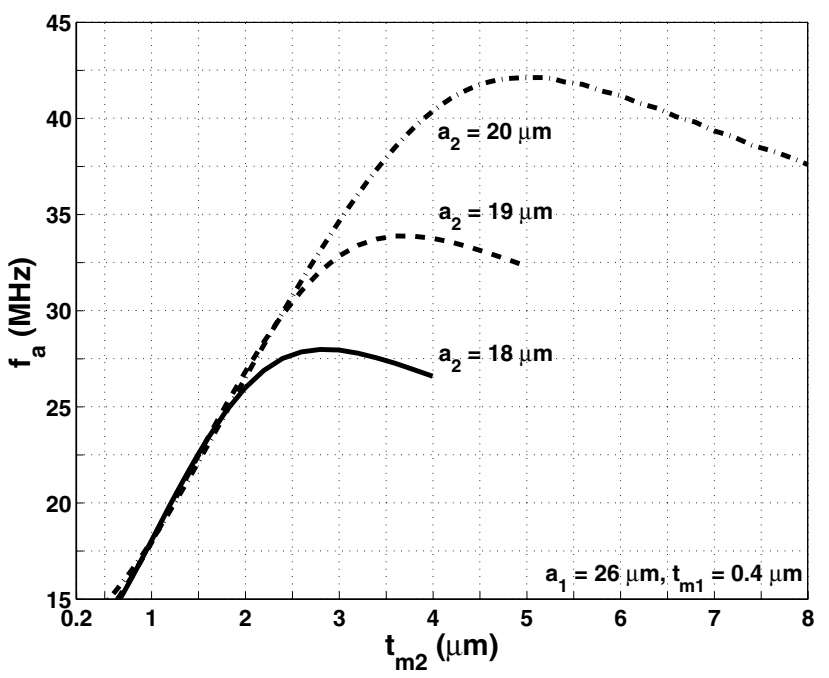

(b) $f_{a}$

Fig. 4. Change of (a) $f_{r}$ and (b) $f_{a}$ with respect to $t_{m 2}$ for various $a_{2}$ values when $a_{1}$ and $t_{m 1}$ are held constant at $26 \mu \mathrm{m}$ and $0.4 \mu \mathrm{m}$.

\section{OPERATION MODES}

In the following, two figures of merit in terms of gainbandwidth product defined in [5] are used to compare the performance of cMUTs with the uniform and nonuniform membranes. The bias voltages for transmit and receive modes are assumed to be 0.45 and 0.9 of the collapse voltage, $V_{c o l}$, respectively. The top electrode is assumed at the bottom of the membrane. For a uniform membrane with an electrode coverage of $\% 70$ of $a$ and a bias set to $0.9 V_{c o l}, V_{c o l}$ and 


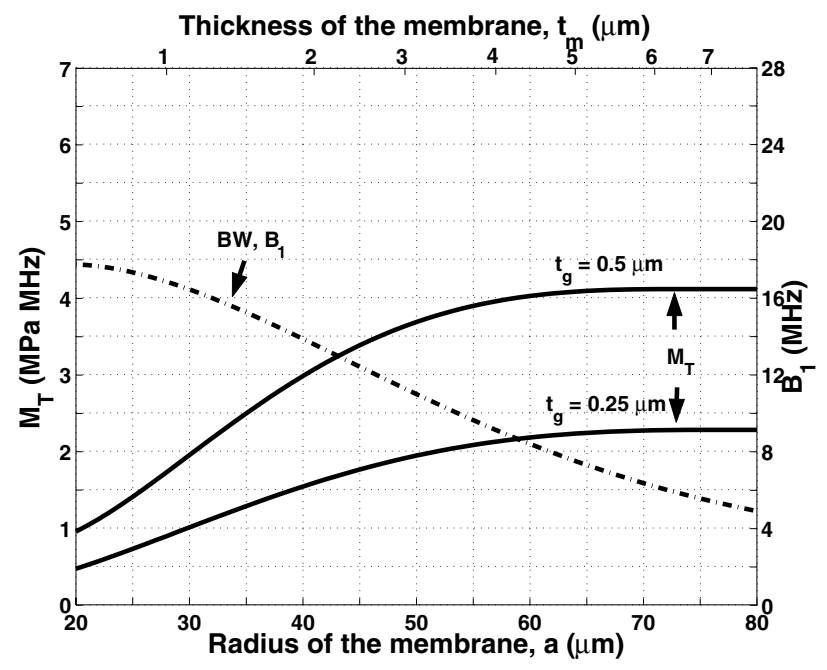

(a) Uniform membrane.

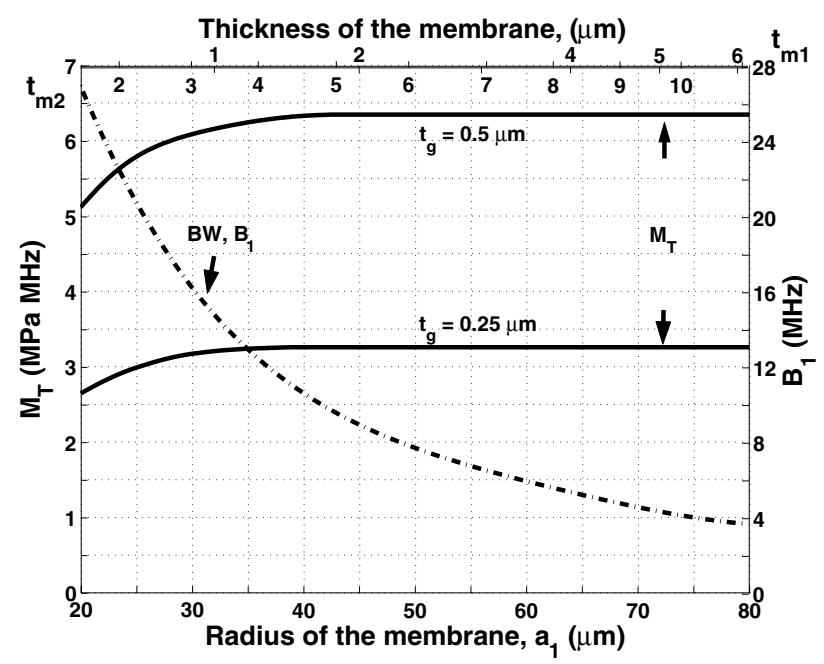

(b) Nonuniform membrane.

Fig. 5. Pressure-bandwidth product, $M_{T}$ (solid) and bandwidth $B_{1}$ (dash-dot), of cMUTs with the (a) uniform membrane (b) nonuniform membrane with respect to the membrane radius having $f_{r}$ of $5.5 \mathrm{MHz}$ for various $t_{g}$.

$n$ do not change, while $C_{O}$ significantly reduces [7]. The same result holds for a nonuniform membrane if the electrode covers only the nonuniform region. For the sake of simplicity, the spurious capacitors are not included in the calculations. To make a fair comparison between the performance of the devices, $f_{r}$ of each device is kept constant. This is achieved by keeping $t_{m} / a^{2}$ constant for a uniform membrane. On the other hand, referring to Fig. 4(a), the situation is quite different for a nonuniform membrane, since for a given $a_{1}$, there are more than one solution for a desired $f_{r}$. In this work, we restrict ourselves to setting $a_{2} / a_{1}$ equal to 0.75 and $t_{m 1} / t_{m}{ }^{1}$ to 0.6 . Since there are two possible $t_{m 2}$ values, we choose the membranes obtained from the second solution, which gives the higher $f_{a}$, thus the possibility of obtaining a higher bandwidth. $f_{r}$ is set to $5.5 \mathrm{MHz}$ for both uniform and nonuniform membranes. The loading medium is assumed to be water $\left(Z_{a}=1.5 \times 10^{6} \mathrm{~kg} / \mathrm{m}^{2} \mathrm{~s}\right)$.

\section{A. Transmission Mode}

During the transmission mode, there is no limitation in terms of the available power. The only limitation is the applied voltage due to the breakdown of the insulator material or the collapse voltage of the membrane. Referring to Fig. 2(a), it is important to maximize the pressure, $P$, at the mechanical side, which is given by $P=F / S$. Let $B_{1}$ be the associated 3-dB bandwidth, then the figure of merit for the transmit mode is defined as [5]:

$$
M_{T}=P B_{1}
$$

While calculating the equivalent circuit parameters, the maximum peak voltage on the electrode is assumed to be 0.9 of the membrane collapse voltage, $V_{c o l}$, and cMUT is biased at

\footnotetext{
${ }^{1} t_{m}$ corresponds to the required membrane thickness if a uniform membrane is constructed with radius $a_{1}$ to resonate at $f_{r}$
}

0.45 of $V_{c o l}$. The higher order harmonics generated during the transmission is neglected.

The change of $M_{T}$ with respect to the membrane radius can be seen in Fig. 5 for both uniform and nonuniform membranes for $t_{g}$ set at 0.25 and $0.5 \mu \mathrm{m} . B_{1}$ is independent of $t_{g}$, whereas $M_{T}$ increases with $t_{g}$ due to the maximum applied voltage, closely related to the $V_{c o l}$. For a uniform membrane, the maximum achievable bandwidth (at $a=20 \mu \mathrm{m}$ ) is $17.7 \mathrm{MHz}$ with a pressure of $27 \mathrm{kPa}$ when $t_{g}=0.25 \mu \mathrm{m}$. As the radius increases, $B_{1}$ decreases whereas $M_{T}$ increases. For small $a$, $Z_{m}$ is negligible compared to the acoustic impedance of the medium; hence $f_{a}$ limits bandwidth. On the other hand, as $a$ increases, $Z_{m}$ begins to increase lowering the bandwidth. A nonuniform membrane configuration can give (at $a_{1}=20 \mu \mathrm{m}$ ) a $B_{1}$ of $27 \mathrm{MHz}$ with a considerably high pressure $(100 \mathrm{kPa}$ when $\left.t_{g}=0.25 \mu \mathrm{m}\right)$. Also for $a_{1}$ greater than $30 \mu \mathrm{m}$, $M_{T}$ remains constant, which gives the possibility of trading between $\mathrm{P}$ and $B_{1}$ without degrading the pressure-bandwidth product. $Z_{m}$ of the nonuniform membrane grows faster than the uniform one, resulting in the more degradation in $B_{1}$ for higher $a$. The higher $n$ and $f_{a}$ compared to uniform membrane is responsible for the superior performance.

\section{B. Receive Mode}

In the receive mode, the input acoustic power is not unlimited. Hence it is important to use as much of the available acoustic power as possible. To obtain the best performance, the acoustic mismatch at the mechanical side (Fig. 2(b)) should be minimized. Similarly, the electrical mismatch at the electrical side should be kept at the minimum. For such a case, the transducer power gain, $G_{T}$, is a fair way of describing the performance. The figure of merit for the receive mode is defined as [5]:

$$
M_{R}=\sqrt{G_{T}} B_{2}
$$




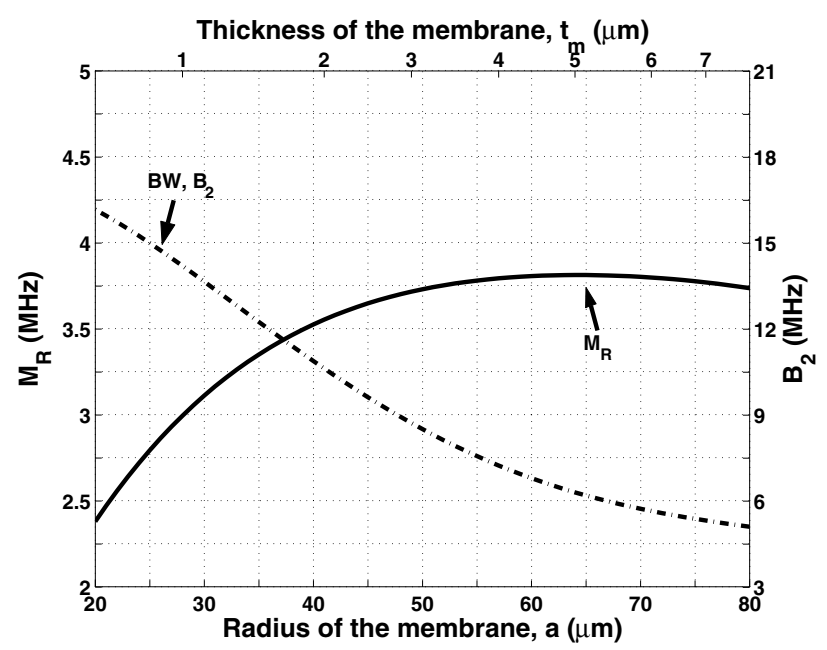

(a) Uniform membrane.

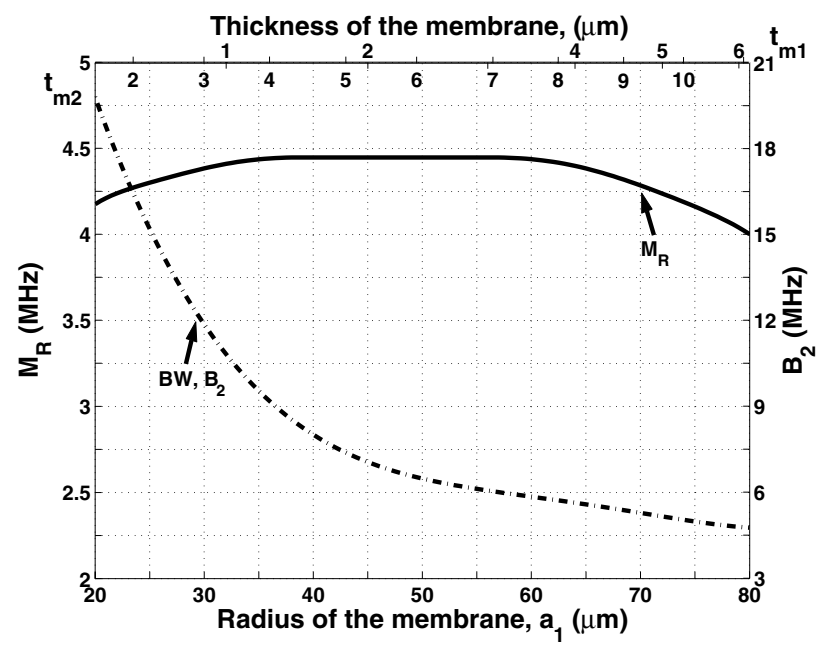

(b) Nonuniform membrane.

Fig. 6. Gain-bandwidth product, $M_{R}$ (solid) and bandwidth $B_{2}$ (dash-dot), of cMUTs with the (a) uniform membrane (b) nonuniform membrane with respect to the membrane radius having $f_{r}$ of $5.5 \mathrm{MHz}$.

where $B_{2}$ is the $3-\mathrm{dB}$ bandwidth of the transducer gain. It is clear that the termination resistance, $R_{S}$, plays a critical role since it affects the reflection coefficient at the electrical side. It is possible to find an $R_{S}$ value, where $M_{R}$ is maximized. Fig. 6 shows the change of $M_{R}$ with respect to the membrane radius, when the electrical side is terminated with the $R_{S}$ value such that $M_{R}$ is maximized. $M_{R}$ and $B_{2}$ are found to be independent of $t_{g}$. For the uniform membrane, the maximum gain-bandwidth product is achieved around $a=60 \mu \mathrm{m}$, which corresponds to $M_{R}=3.8 \mathrm{MHz}$ with $B_{2}$ equal to $6.8 \mathrm{MHz}$ (hence $G_{T}$ equal to $-5 \mathrm{~dB}$ ). $B_{2}$ increases as $a$ gets smaller, since $Z_{m}$ is small compared to the impedance of the loading medium, water. On the other hand, a nonuniform membrane gives an $M_{R}$ of $4.44 \mathrm{MHz}$ around $a_{1}=50 \mu \mathrm{m}$ with a $B_{2}$ of $6.5 \mathrm{MHz}$. Again for small $a_{2}$ value, $B_{2}$ increases. The variation of $M_{R}$ is within $\% 10$ of the maximum, making an efficient trade-off between gain and bandwidth possible. For $a_{2}=20 \mu \mathrm{m}, B_{2}$ and $G_{T}$ are equal to $19.8 \mathrm{Mhz}$ and $-13.5 \mathrm{~dB}$, respectively. On the contrary, a uniform membrane can achieve such a bandwidth with a very small membrane radius, with a much lower gain.

\section{CONCLUSIONS}

cMUTs offer a high bandwidth in the high impedance media at the expense of low gain due to their low turns ratio, $n$. The bandwidth of the device is limited by the second resonance of the membrane, for both transmission and reception. In this work, it is shown that placing a mass at the central part of the membrane shifts the antiresonance frequency to higher values in addition to increasing the turns ratio. The performance comparison of cMUTs with uniform and nonuniform membranes with reduced electrode sizes are made using the gainbandwidth product. cMUTs with the nonuniform membranes are found to be superior compared to the uniform ones. It is also shown that it is possible to trade the gain with bandwidth without degrading the product of both.

\section{REFERENCES}

[1] G. G. Yaralioglu, M. H. Badi, A. S. Ergun, and B. T. Khuri-Yakub, "Improved equivalent circuit and finite element method modeling of capacitive micromachined ultrasonic transducer," in Proc. of IEEE Ultrasonics Symposium, 2003, pp. $469-472$.

[2] Y. Huang, A. S. Ergun, Edward, M. H. Badi, and B. T. Khuri-Yakub, "Fabricating capacitive micromachined ultrasonic transducers with waferbonding technology," Journal of Micromechanical Systems, vol. 12, no. 2, pp. $128-137,2003$.

[3] Y. Huang, , E. Hæggström, X. Zhuang, A. S. Ergun, and B. T. KhuriYakub, "Optimized membrane configuration improves cMUT performance," in Proc. of IEEE Ultrasonics Symposium, 2004, pp. 505 - 508

[4] J. Knight and F. Degertekin, "Capacitive micromachined ultrasonic transducers for forward looking intravascular imaging arrays." in Proc. of IEEE Ultrasonics Symposium, 2002, pp. 1079-1082.

[5] S. Olcum, M. N. Senlik, and A. Atalar, "Optimization of the gainbandwidth product of capacitive micromachined ultrasonic transducer," IEEE Transactions on Ultrasonics, Ferroelectrics, and Frequency Control, to be published.

[6] A. Bozkurt, I. Ladabaum, A. Atalar, and B. T. Khuri-Yakub, "Theory and analysis of electrode size optimization for capacitive microfabricated ultrasonic transducers," IEEE Transactions on Ultrasonics, Ferroelectrics, and Frequency Control, vol. 46, no. 6, pp. 1364 - 1374, 1999.

[7] M. N. Senlik, "Nonuniform membranes in capacitive micromachined ultrasonic transducers," Master's thesis, Bilkent University, 2005. [Online]. Available: http://www.thesis.bilkent.edu.tr/0002776.pdf 